Journal Club

Editor's Note: These short reviews of recent JNeurosci articles, written exclusively by students or postdoctoral fellows, summarize the important findings of the paper and provide additional insight and commentary. If the authors of the highlighted article have written a response to the Journal Club, the response can be found by viewing the Journal Club at www.jneurosci.org. For more information on the format, review process, and purpose of Journal Club articles, please see https://www.jneurosci.org/content/ jneurosci-journal-club.

\title{
Female-Specific Effects of CGRP Suggest Limited Efficacy of New Migraine Treatments in Males
}

\author{
다rancie Moehring and ๑Katelyn E. Sadler \\ Department of Cell Biology, Neurobiology and Anatomy, Medical College of Wisconsin, Milwaukee, Wisconsin 53226 \\ Review of Avona et al.
}

Skepticism of preclinical analgesic development has existed since the failed and discontinued clinical trials of substance $\mathrm{P}$ receptor [neurokinin 1 (NK1)] antagonists (Hill, 2000) and TRPV1 antagonists, respectively (Gavva et al., 2008). Scrutiny of preclinical pain research has also increased in light of the current opioid epidemic. Our field's limited translational success has likely resulted from many factors including less-than-rigorous experimental design and execution, crossspecies target expression differences, and animal pain model relevance, including the disproportionate use of male animals and tissue samples in preclinical testing (Mogil, 2009; Clayton and Collins, 2014). Given these issues, the recent FDA approval of three monoclonal antibodies (erenumab, fremanezumab, and galcanezumab) that target calcitonin generelated peptide (CGRP) or its cognate receptor for treating migraine is a significant achievement for both preclinical and clinical pain research. These compounds are the first treatments to be approved for migraine attacks since triptan compounds entered the market in the early 1990s.

\footnotetext{
Received May 30, 2019; revised Sept. 12, 2019; accepted Sept. 21, 2019. We thank Dr. Cheryl Stucky for insightful edits and Neil Smith for digital artwork production.

The authors declare no competing financial interests.

Correspondence should be addressed to Katelyn E. Sadler at ksadler@mcw.edu.

https://doi.org/10.1523/JNEUROSCI.1254-19.2019

Copyright $\odot 2019$ the authors
}

Despite the approval of these compounds, questions regarding sex-specific analgesic efficacy exist because male rodents/tissue were used in many preclinical studies of this female predominant pain condition. To address this issue, a recent Journal of Neuroscience article by Avona et al. (2019) investigated the sex-specific effects of CGRP in rodent migraine-like behaviors. Avona et al. (2019) injected CGRP directly onto the dura, the outermost meningeal layer that encapsulates the brain, of male and female mice and rats, and then measured facial mechanical sensitivity and spontaneous pain-like behavior using grimace scoring. Female rodents developed facial mechanical hypersensitivity after dural application of CGRP doses as low as $1 \mathrm{pg}$; male rodents did not develop facial hypersensitivity at any CGRP dose tested (up to $3.8 \mu \mathrm{g}$ ). Female mice also exhibited higher grimace scores after dural CGRP injections; male mice never displayed altered facial parameters after CGRP infusion. Interestingly, intraplantar injections of CGRP also produced hindpaw mechanical hypersensitivity in female mice but not males, suggesting that the sex-specific effects of CGRP are not limited to dura. Based on these data, anti-CGRP therapies are expected to decrease widespread pain-like behaviors in female subjects, but not males. However, in previous studies, CGRP antagonists successfully decreased complete Freud's adjuvant, osteoarthritis, and hind- paw plantar incision pain-like behaviors in male rodents (Hirsch et al., 2013; Bullock et al., 2014; Cowie et al., 2018). In these models, circulating CGRP levels may be significantly higher than those used by Avona et al. (2019), and thus, CGRP antagonist analgesia may be achievable in both sexes.

The exact mechanisms through which CGRP drives migraine-like behaviors are unknown, but primary sensory afferents, which are required for the transmission of noxious peripheral stimuli and initiation of pain-like behaviors, are a convergent site for CGRP-related activities (Fig. 1). CGRP increases excitability of peripheral sensory neurons isolated from male dorsal root ganglia (DRG) by increasing activity of protein kinase A (PKA) and protein kinase $\mathrm{C}(\mathrm{PKC})$, subsequent release of calcium from intracellular stores, and sensitization of voltage-dependent calcium and sodium currents (Natura et al., 2005). Nonetheless, extracellular application of CGRP alone does not initiate spiking in DRG neurons. Similarly, when CGRP is systemically, intravenously, or topically applied to the dura, ongoing discharge rates of trigeminal ganglia (TG) A $\delta$ and C fiber neurons do not change (Levy et al., 2005); again, these experiments were only completed in male animals. The effects of CGRP on female TG or DRG neuronal excitability are, to our knowledge, unknown, but might contribute to the sex-specific behavioral effects of this neuropeptide. 
In addition to general cell excitability, CGRPmightsensitizeligand-or mechanicallygated ion channels in TG neurons. It is hypothesized (Levy et al., 2005) that CGRP-induced vasodilation increases the mechanical forces within dural blood vessels, and thus may drive TG neuronal activity via mechanically-gated (e.g., Piezo2) or mechanically-relevant (e.g., TRPA1, TRPV4) ion channels (Huang et al., 2012; Ranade et al., 2015). Experiments performed by Levy et al. (2005) did not support this hypothesis, because systemic, intravenous, or direct dural application of CGRP did not decrease mechanical thresholds or increase mechanically-induced firing frequencies of TG $\mathrm{A} \delta$ and $\mathrm{C}$ fiber neurons. Unfortunately, however, these in vivo recordings were only completed in male rats and only tested a single CGRP dose for each administration route. To our knowledge, CGRP-induced changes in mechanical sensitivity have never been investigated in TG or DRG neurons isolated from female rodents. Investigations of this hypothesized sensitization via patch-clamp and/or single-fiber recordings would provide valuable insight into the broader roles of CGRP in the mechanical hypersensitivity that develops in chronic pain conditions, many of which are characterized by neurogenic inflammation and local release of CGRP (Vincent et al., 2013; Schou et al., 2017; Cowie et al., 2018).

In addition to activation via mechanical stimuli, dural afferents may be activated by chemical stimuli during migraine attacks. Avona et al. (2019) reported that dural administration of a subthreshold chemical stimulus (e.g., synthetic intestinal fluid $\mathrm{pH} 7.0$ or nitric oxide donor) elicited facial mechanical hypersensitivity in female rats that had recovered from previous dural applications of CGRP (Avona et al., 2019). It is possible that the initial application of CGRP induced the release of NO from CGRP-responsive vascular endothelial cells (Gray and Marshall, 1992) and/or a mixture of cytokines, histamine, and other proinflammatory mediators from CGRP-responsive mast cells (Theoharides et al., 2005). Incubation of dural $\mathrm{C}$ and $\mathrm{A} \delta$ fibers in this proinflammatory microenvironment might lead to the direct activation or sensitization of various channels expressed on the afferents, including transient receptor potential vanilloid 1 (TRPV1). When small diameter neurons expressing TRPV1 are activated by endogenous channel ligands, including anandamide and protons, CGRP is released from the peripheral terminals through a process known as neurogenic in-
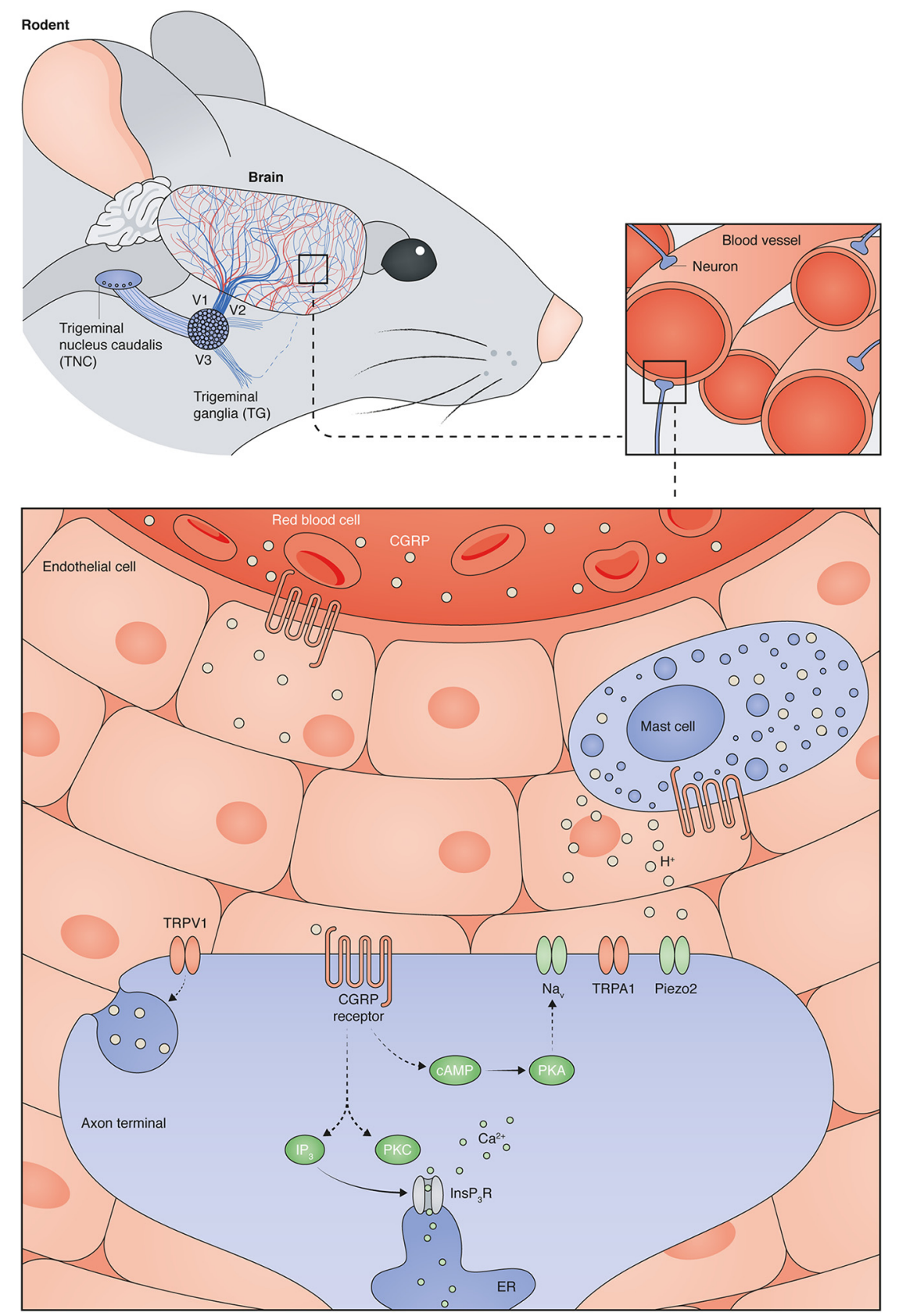

Figure 1. Potential mechanisms through which CGRP might modulate dural afferent activity. The rodent dura is a highly vascularized and innervated tissue. Many dural afferents, the cell bodies of which are located in the trigeminal ganglia, terminate in close proximity to blood vessel endothelial cells. Circulating CGRP and GRP released from mast cells activates CGRP receptors. In neurons, CGRP receptor activity leads to (1) adenylyl cyclase activation, subsequent increases in CAMP, and protein kinase A-induced sensitization of plasma membrane channels (e.g., voltage-gated sodium channels $\left.\left(\mathrm{Na}_{\mathrm{v}}\right)\right)$ or $(2)$ phospholipase-Cmediated synthesis of $I P_{3}$ and DAG, subsequent activation of protein kinase $C$ and release of intracellular calcium stores. Collectively, these activities can increase neuronal excitability. Specific activation of TRPV1-expressing dural afferents can lead to peripheral release of CGRP, which can perpetuate CGRP signaling in nearby neurons and non-neuronal cells.

flammation (Akerman et al., 2004; Meng et al., 2009). Neuronal release of CGRP increases local tone, thus further amplifying CGRP-mediated activities in all cells expressing the cognate receptor (i.e., endothelial and mast cells). Proinflammatory mediators released by these cells additionally sensitize TRPV1 so that lower agonist concentrations are required for channel opening, thus perpetuating a feedforward CGRP release process. CGRP-induced sensitization of TRPV1 may also contribute to the mechanical hypersensitivity observed in females rodents in these studies (Avona et al., 2019). Although not intrinsically mechanically sensitive, TRPV1 contributes to behavioral mechanical hypersensitivity in many proinflammatory conditions (Mickle 
et al., 2015; Watanabe et al., 2015; Sadler et al., 2018); the exact mechanisms underlying this novel channel activity are unclear at present. To our knowledge, TRPV1 sensitization and TRPV1-mediated CGRP release have only been assessed in male rodents, and thus, additional studies should be completed in female rodents to determine whether equivalent processes happen in both sexes. If they do, this might explain the female-specific priming effect reported by Avona et al. (2019).

It is notable that Avona et al. (2019) never detected CGRP-induced pain-like behaviors in male mice or rats; these data conflict with male grimace scores previously reported by Rea et al. (2018). One discrepancy between these two studies is the dose of CGRP: Rea et al. (2018) used $>10$-fold higher dose than Avona et al. (2019). Additionally, Rea et al. (2018) administered CGRP via an intraperitoneal injection, whereas Avona et al. (2019) applied CGRP directly onto the dura. Without testing additional CGRP doses in male mice, including a dose that elicits mechanical hypersensitivity and higher grimace scores after dural application, it is difficult to assess whether the sex differences in CGRP-mediated pain-like behaviors found by Avona et al. (2019) result from decreased expression or sensitivity of CGRP receptor complexes in male TG neurons or increased basal levels of circulating CGRP in male mice.

An additional critique of this paper, and the broader migraine field, is the limited behavioral assays used to study migraine-like pain. Avona et al. (2019) used both reflexive (e.g., von Frey) and spontaneous (e.g., grimace) behavioral measures in this report, but as many migraineurs will note, the experience of a migraine is not limited to, or sometimes even characterized by, similar symptoms. During a migraine attack, patients often report nausea, photophobia, osmophobia, and phonophobia. In the study by Rea et al. (2018), peripheral CGRP administration induced light aversion in both male and female mice; again, these behavioral similarities between sexes may result from the dose or application route of CGRP. Regardless, we believe that additional behavioral tests (e.g., sucrose preference testing, open-field activity, ability of anti-CGRP therapies to block CGRPinduced place aversion, etc.) should be performed to further characterize sexspecific migraine-like behaviors as they relate to CGRP signaling.

Based on the data presented by Avona et al. (2019), low levels of CGRP drive migraine-like behaviors in female rodents only. Although implicated in the pathology of many acute and chronic pain conditions, sex-specific CGRP behavioral effects had not been well described before this report. As outlined above, this neuropeptide may differentially drive pain-like behaviors in each sex through its direct or indirect sensitization of numerous molecular targets. Continued investigations into these processes will allow for tailored therapy design and application, the latter of which should be strongly considered for anti-CGRP therapies.

\section{References}

Akerman S, Kaube H, Goadsby PJ (2004) Anandamide acts as a vasodilator of dural blood vessels in vivo by activating TRPV1 receptors. Br J Pharmacol 142:1354-1360.

Avona A, Burgos-Vega C, Burton MD, Akopian AN, Price TJ, Dussor G (2019) Dural calcitonin gene-related peptide produces femalespecific responses in rodent migraine models. J Neurosci 39:4323-4331.

Bullock CM, Wookey P, Bennett A, Mobasheri A, Dickerson I, Kelly S (2014) Peripheral calcitonin gene-related peptide receptor activation and mechanical sensitization of the joint in rat models of osteoarthritis pain. Arthritis Rheumatol 66:2188-2200.

Clayton JA, Collins FS (2014) NIH to balance sex in cell and animal studies. Nature 509: 282-283.

Cowie AM, Moehring F, O’Hara C, Stucky CL (2018) Optogenetic inhibition of CGRP $\alpha$ sensory neurons reveals their distinct roles in neuropathic and incisional pain. J Neurosci 38:5807-5825.

Gavva NR, Treanor JJ, Garami A, Fang L, Surapaneni S, Akrami A, Alvarez F, Bak A, Darling M, Gore A, Jang GR, Kesslak JP, Ni L, Norman MH, Palluconi G, Rose MJ, Salfi M, Tan E, Romanovsky AA, Banfield C, Davar G (2008) Pharmacological blockade of the vanilloid receptor TRPV1 elicits marked hyperthermia in humans. Pain 136:202-210.

Gray DW, Marshall I (1992) Human $\alpha$-calcitonin gene-related peptide stimulates adenylate cyclase and guanylate cyclase and relaxes rat thoracic aorta by releasing nitric oxide. $\mathrm{Br} \mathrm{J}$ Pharmacol 107:691-696.

Hill R (2000) NK1 (substance P) receptor antagonists: why are they not analgesic in humans? Trends Pharmacol Sci 21:244-246.

Hirsch S, Corradini L, Just S, Arndt K, Doods H (2013) The CGRP receptor antagonist BIBN4096BS peripherally alleviates inflammatory pain in rats. Pain 154:700-707.
Huang D, Li S, Dhaka A, Story GM, Cao YQ (2012) Expression of the transient receptor potential channels TRPV1, TRPA1 and TRPM8 in mouse trigeminal primary afferent neurons innervating the dura. Mol Pain 8:66.

Levy D, Burstein R, Strassman AM (2005) Calcitonin gene-related peptide does not excite or sensitize meningeal nociceptors: implications for the pathophysiology of migraine. Ann Neurol 58:698-705.

Meng J, Ovsepian SV, Wang J, Pickering M, Sasse A, Aoki KR, Lawrence GW, Dolly JO (2009) Activation of TRPV1 mediates calcitonin gene-related peptide release, which excites trigeminal sensory neurons and is attenuated by a retargeted botulinum toxin with antinociceptive potential. J Neurosci 29:49814992.

Mickle AD, Shepherd AJ, Loo L, Mohapatra DP (2015) Induction of thermal and mechanical hypersensitivity by parathyroid hormonerelated peptide through upregulation of TRPV1 function and trafficking. Pain 156: $1620-1636$.

Mogil JS (2009) Animal models of pain: progress and challenges. Nat Rev Neurosci 10: 283-294.

Natura G, von Banchet GS, Schaible HG (2005) Calcitonin gene-related peptide enhances TTX-resistant sodium currents in cultured dorsal root ganglion neurons from adult rats. Pain 116:194-204.

Ranade SS, Syeda R, Patapoutian A (2015) Mechanically activated ion channels. Neuron 87:1162-1179.

Rea BJ, Wattiez AS, Waite JS, Castonguay WC, Schmidt CM, Fairbanks AM, Robertson BR, Brown CJ, Mason BN, Moldovan-Loomis MC, Garcia-Martinez LF, Poolman P, Ledolter J, Kardon RH, Sowers LP, Russo AF (2018) Peripherally administered calcitonin gene-related peptide induces spontaneous pain in mice: implications for migraine. Pain 159:2306-2317.

Sadler KE, Zappia KJ, O'Hara CL, Langer SN, Weyer AD, Hillery CA, Stucky CL (2018) Chemokine (c-c motif) receptor 2 mediates mechanical and cold hypersensitivity in sickle cell disease mice. Pain 159:1652-1663.

Schou WS, Ashina S, Amin FM, Goadsby PJ, Ashina M (2017) Calcitonin gene-related peptide and pain: a systematic review. J Headache Pain 18:34.

Theoharides TC, Donelan J, Kandere-Grzybowska K, Konstantinidou A (2005) The role of mast cells in migraine pathophysiology. Brain Res Rev 49:65-76.

Vincent L, Vang D, Nguyen J, Gupta M, Luk K, Ericson ME, Simone DA, Gupta K (2013) Mast cell activation contributes to sickle cell pathobiology and pain in mice. Blood 122: 1853-1862.

Watanabe M, Ueda T, Shibata Y, Kumamoto N, Ugawa S (2015) The role of TRPV1 channels in carrageenan-induced mechanical hyperalgesia in mice. Neuroreport 26:173-178. 